\title{
RADIACIÓN ELECTROMAGNÉTICA, LEUCEMIA INFANTIL Y REGULACIÓN
}

Electromagnetic radiation, childhood leukemia and regulation

\author{
Jesús Leonardo SOTO SUMUANO ${ }^{1 *}$, Emmanuel ABUNDIS GUTIÉRREZ ${ }^{2}$, \\ José Alberto TLACUILO-PARRA ${ }^{3}$, Roberto Francisco GARIBALDI COVARRUBIAS ${ }^{3}$ y \\ Hugo ROMO RUBIO ${ }^{4}$
}

${ }^{1}$ Universidad de Guadalajara, Periférico Norte 799, Módulo L 204, Núcleo Universitario Los Belenes, 45100 Zapopan, Jalisco México

${ }^{2}$ Sociedad Mexicana para la Protección de la Radiación no Ionizante, Club Nacional 665, Col. Auditorio, 45180 Zapopan, Jalisco, México

${ }^{3}$ Instituto Mexicano del Seguro Social, UMAE Hospital de Pediatría CMNO, Belisario Domínguez 735, Col. Independencia, 44340 Guadalajara, Jalisco, México

${ }^{4}$ Hospital Civil de Guadalajara, Salvador Quevedo y Zubieta 750, Col. Independencia Oriente, 44340 Guadalajara, Jalisco, México

*Autor para correspondencia: leonardo.1soto@gmail.com

(Recibido: diciembre 2018; aceptado: agosto 2019)

Palabras clave: salud pública, contaminación electromagnética, frecuencia extremadamente baja, radio frecuencia, Norma Oficial Mexicana

\section{RESUMEN}

Desde 2002 se ha reconocido que la exposición a la radiación electromagnética no ionizante (RNI) en el rango de las frecuencias extremadamente bajas (FEB) constituye un riesgo para la salud. La Organización Mundial de la Salud y la Agencia Internacional para la Investigación sobre el Cáncer clasificaron su exposición en humanos dentro del grupo 2B como posible carcinógeno. Posteriormente, en 2011 se agregó a esta clasificación a las radiofrecuencias (RF), incluyendo las microondas ampliamente utilizadas en la oferta de servicios inalámbricos. Como consecuencia de ello, hubo un incremento a nivel mundial de actividades científicas, sociales y de gobierno para estudiar estas emisiones y encontrar medidas preventivas que permitieran reducir este factor de riesgo a la salud mediante políticas de baja exposición en la población en general. En este escenario, México tiene la oportunidad de actualizar y generar nuevas normas oficiales mexicanas que propicien por una parte regular los niveles máximos permitidos de exposición a RNI y, por la otra, realizar vigilancia y control de los efectos a la salud. El presente artículo aborda las características de las RNI, analiza el marco regulatorio internacional y nacional, presenta los resultados obtenidos de la medición de RNI intradomiciliarias en el rango de FEB y RF de pacientes pediátricos con leucemia aguda en el área metropolitana de Guadalajara; por último, propone una serie de recomendaciones y acciones.

Key words: public health, electromagnetic pollution, extremely low frequency, radio frequency, Official Mexican Standards 


\begin{abstract}
Since 2002, the World Health Organization has recognized non-ionizing electromagnetic radiation (NIR) in the extremely low frequency range (ELF) to constitute a health risk and has placed human exposure to ELF within the group 2B as a possible human carcinogen. Subsequently, in 2011, radio frequencies (RF) used in wireless telecommunications were added to this classification. As a result, there was a worldwide increase in scientific, social and government activities to study these emissions and find preventive measures to reduce exposure to the general population. Mexico, faced with this scenario, has the opportunity to update and generate new Official Mexican Standards (NOM), which would allow, on one hand, the regulation of the maximum permitted levels of NIR and, on the other hand, to monitor and control their health effects. This article addresses the characteristics of NIR, analyzes the international and national environmental regulatory framework, presents the results obtained from the measurement of NIR in ELF and intra-domiciliary RF of pediatric patients with acute leukemia in the metropolitan area of Guadalajara and finally, it proposes a series of recommendations and actions.
\end{abstract}

\section{INTRODUCCIÓN}

La radiación electromagnética se define como el flujo saliente de energía de una fuente o emisor en forma de ondas electromagnéticas. Se clasifica en ionizante (RI) y no ionizante (RNI). El empleo de las radiofrecuencias (RF) para la transmisión de información a distancia y el uso de energía eléctrica en todas las actividades del ser humano, ha dado lugar a una presencia permanente de RNI en el medio ambiente urbano. La diferencia fundamental entre la RNI y la RI es que esta última posee suficiente energía para ionizar la materia al situarse en un rango de frecuencia elevado (mayor a $10^{15} \mathrm{~Hz}$ ), la cual se considera dañina para la salud por ser causa de múltiples enfermedades, entre otras el cáncer (Fig. 1). Por su parte, las RNI no están asociadas con energía suficiente para producir ionización, ya que no generan iones y sus efectos térmicos sólo son percibidos por el ser humano en el rango de las microondas (ICNIRP 2009, Cabañas 2017). Esto no significa que la radiación no ionizante esté exenta de ocasionar efectos negativos en la salud. Como referencia, se menciona que en 2002 la Agencia Internacional de Investigación sobre el Cáncer (IARC, por sus siglas en inglés) evaluó los riesgos de la RNI en el rango de frecuencias extremadamente bajas (FEB), clasificándola en el grupo 2B como agente posiblemente carcinogénico para el ser humano (IARC 2002). Posteriormente, en 2011 la misma IARC evaluó el riesgo de cáncer asociado con la exposición a RNI en el rango de radiofrecuencias (RF), la cual catalogó de igual manera como posible carcinogénico 2B (IARC 2011).
Las anteriores afirmaciones generaron una gran preocupación en el mundo médico por los efectos adversos a la salud que pudiere causar el aumento de exposición a RNI, a la que, sin lugar a duda, todos los seres humanos estamos expuestos por el desarrollo exponencial de nuevos dispositivos y servicios de acceso inalámbrico de uso común, motivo por el cual se le considera una de las posibles razones de aparición temprana de enfermedades y trastornos en la salud de los niños (Kundi 2012).

La RNI de interés en este trabajo es la producida por las ondas electromagnéticas en cuyas frecuencias se ubican las aplicaciones y servicios más utilizados por el ser humano, específicamente la producida por los campos magnéticos de las FEB cuyo rango va de los 3 hasta los $3000 \mathrm{~Hz}$, y por la radiación producida en el rango de las RF, cuyo rango va de los 30 hasta los $300 \mathrm{GHz}$ (IEEE 1992) (Fig. 1).

\section{MARCO REGULATORIO INTERNACIONAL}

En 1974, la Asociación Internacional para la Protección contra la Radiación (IRPA, por sus siglas en inglés) formó un grupo de trabajo para examinar los problemas de salud generados por las RNI. En el congreso de la IRPA en París, realizado en 1977, este grupo se convirtió en el Comité Internacional para las Radiaciones No-Ionizantes (INIRC, por sus siglas en inglés) que, en cooperación con la División de Salud Ambiental de la Organización Mundial de la Salud (OMS), desarrolló información documentada sobre criterios de salud y su relación con las RNI, como parte del programa de criterios de salud ambiental 


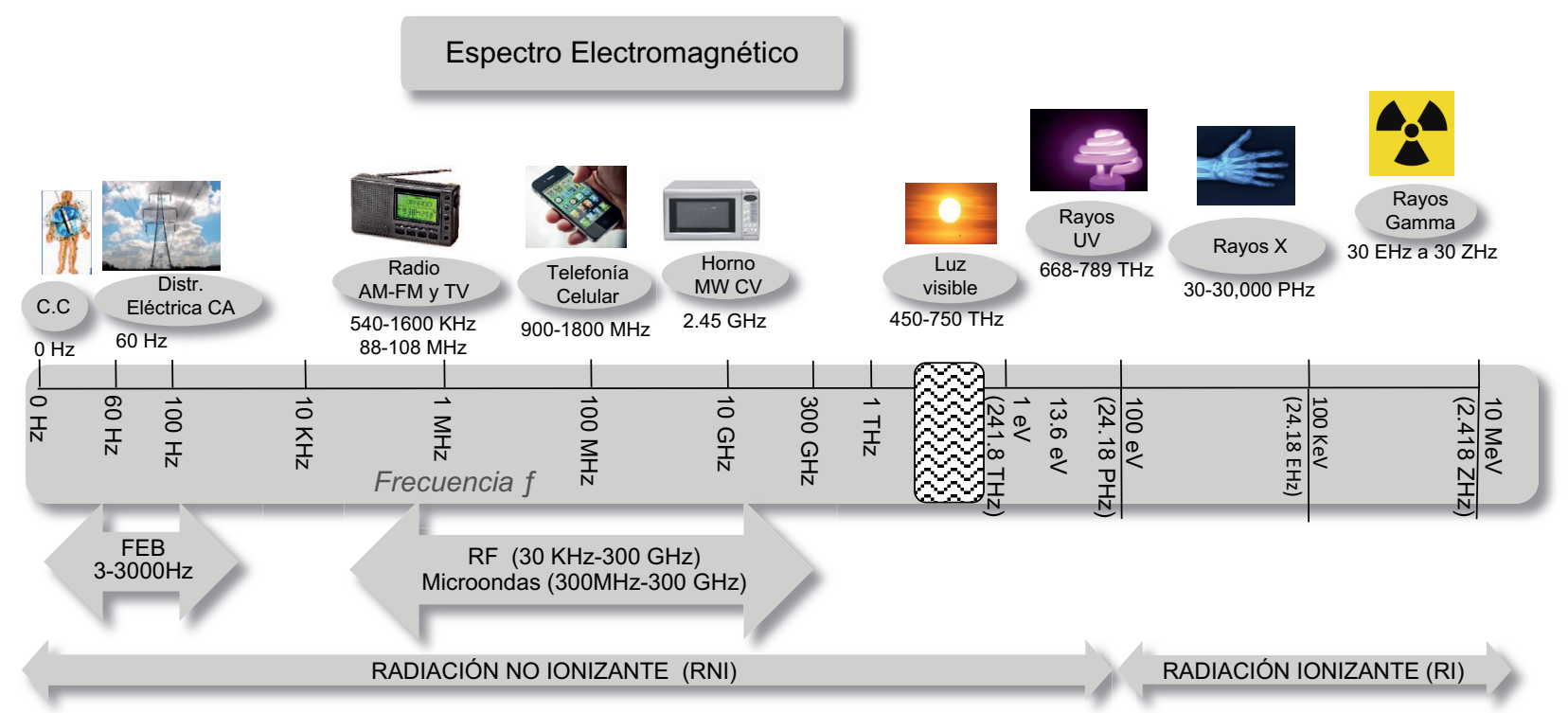

Fig. 1. Espectro electromagnético clasificado por servicios, rangos de frecuencia y tipos de radiación

de la OMS, auspiciado por el Programa de Naciones Unidas para el Ambiente (UNEP, por sus siglas en inglés). En 1992, la Comisión Internacional para la Protección de la Radiación No Ionizante (ICNIRP, por sus siglas en inglés) fue constituida como una comisión independiente para continuar el trabajo del INIRC y la IRPA. La ICNIRP se define como una organización no gubernamental sin ánimo de lucro, integrada por un grupo de científicos reconocida por la OMS, que emite recomendaciones no vinculantes (ICNIRP 2010).

En Estados Unidos, la Comisión Federal de Comunicaciones (FCC, por sus siglas en inglés) estableció en 1996 una regulación de la exposición humana a campos electromagnéticos en el rango de las RF, cuyo nivel máximo de densidad de potencia no debe superar los $10 \mathrm{~W} / \mathrm{m}^{2}$. Dicha norma se encuentra en revisión desde 2015 debido al crecimiento exponencial registrado en las emisiones desde hace 10 años (FCC-OET 1997).

En 1998, la ICNIRP desarrolló una serie de recomendaciones de carácter no obligatorio que reconocían sólo el efecto térmico de las emisiones, en las que se sugería un máximo de $10 \mathrm{~W} / \mathrm{m}^{2}$, el cual posteriormente fue actualizado a $5 \mathrm{~W} / \mathrm{m}^{2}$ (ICNIRP 2010). Países como Nueva Zelanda han propuesto rangos máximos de $0.01 \mathrm{~W} / \mathrm{m}^{2}$, Suiza de $0.05 \mathrm{~W} / \mathrm{m}^{2}$ e Italia de $0.1 \mathrm{~W} / \mathrm{m}^{2}$, muy por debajo del límite establecido por EUA en zonas de alto riesgo como hospitales y escuelas. Otros países, como China y Rusia, tienen límites tan diferenciados respecto a EUA que suelen confundir a los demás países u organizaciones, generando dudas sobre los efectos de las emisiones (CS 1999).

Cabe señalar que a la fecha la ICNIRP no ha actualizado sus recomendaciones y esta situación ha ocasionado que países sensibles al seguimiento de las investigaciones científicas sobre los efectos en la salud de este tipo de radiaciones, hayan hecho ajustes en el máximo nivel permitido con el objetivo de bajar el riesgo e incrementar los rangos de prevención. A lo antes mencionado, se añade que los límites de la ICNIRP atienden sólo al concepto de efecto térmico de exposición a las RNI, mientras que las nuevas propuestas plantean tomar en cuenta el concepto de efecto no térmico, debido principalmente a estudios que muestran la existencia de efectos biológicos no térmicos adversos a la salud (Singh y Kapoor 2014).

\section{MARCO REGULATORIO EN MÉXICO}

En el transcurso de los últimos 20 años, la industria de las telecomunicaciones en México se convirtió en área estratégica y vital para el desarrollo del país por su oferta de servicios y aplicaciones, además de promover e incentivar un mercado más competitivo con las redes generadas. En 2014 la política de crecimiento por el acceso, promovida por el gobierno federal, lanzó la denominada Estrategia Digital Nacional, que incluyó la reforma a la Ley Federal de Telecomunicaciones y Radiodifusión y programas tales como "México conectado", cuyo objetivo 
consistió en llevar internet de alta velocidad a todo el país (SCT 2017).

Sin embargo, así como esas tecnologías, equipos y dispositivos que reciben y emiten RNI han acarreado muchos beneficios, igualmente han generado la proliferación de agentes contaminantes "posiblemente cancerígenos para el humano". Al conjunto de RNI generadas por la infraestructura eléctrica, las telecomunicaciones, los equipos de uso doméstico e industrial, y el uso masivo de nuevas aplicaciones inalámbricas que puede afectar al cuerpo humano, se le ha denominado "contaminación electromagnética" o "electropolución". Este tipo de contaminación es reconocida como un contaminante en la Ley General de Equilibrio Ecológico y Protección Ambiental (LGEEPA), artículo 5, fracción $\mathrm{XV}$, que establece textualmente: "La regulación de la prevención de la contaminación ambiental originada por ruido, vibraciones, energía térmica, lumínica, radiaciones electromagnéticas y olores perjudiciales para el equilibrio ecológico y el ambiente" (CDDHCU 2018c).

La Constitución Política de los Estados Unidos Mexicanos establece en su artículo $4^{\circ}$, cuarto párrafo, que toda persona tiene derecho a la protección de la salud y, particularmente: "a un medio ambiente sano para su desarrollo y bienestar. El Estado garantizará el respeto a este derecho. El daño y deterioro ambiental generará responsabilidad para quien lo provoque en términos de lo dispuesto por la ley" (CDDHCU 2018a). Adicionalmente, el artículo 5, fracción V, de la Ley General del Equilibrio Ecológico y la Protección al Ambiente (LEGEEPA) establece que es facultad del gobierno federal "La expedición de las normas oficiales mexicanas y la vigilancia de su cumplimiento en las materias previstas en esta Ley"; sin embargo, a la fecha no existe un proceso de creación de NOM que regule la emisión de este contaminante y vigile su cumplimiento (CDDHCU 2018c).

La Ley General de Salud (LGS) tiene como objetivos: la prevención y control de los efectos nocivos de los factores ambientales en la salud del hombre (artículo 3, fracción XIII); la salud ocupacional y el saneamiento básico (artículo 3, fracción XIV); el establecimiento de normas, la adopción de medidas y la realización de acciones tendientes a proteger la salud humana ante los riesgos y daños dependientes de las condiciones del ambiente (artículo 116), y el establecimiento de valores de concentración máxima de contaminantes en el ambiente permisibles para el ser humano (artículo 118, fracción I). Aunado a lo anterior, la LGS le confiere a la investigación en salud un importante papel para lograr varios objetivos, entre ellos el "conocimiento y control de los efectos nocivos del ambiente en la salud" (artículo 96, fracción IV) (CDDHCU 2018d).

La LGS ha sido objeto de varias reformas, destacando la del 5 de julio de 2001 que dio origen a la creación de la Comisión Federal para la Protección contra Riesgos Sanitarios (COFEPRIS) como órgano desconcentrado de la Secretaría de Salud (SSA). En esa reforma se determinó que las materias de salubridad general descritas líneas arriba serán ejercidas por la SSA a través de la COFEPRIS, que se encargará de elaborar y expedir las normas oficiales mexicanas materia de la Ley (artículo 17 bis). En el esquema jurídico de la SSA, a la COFEPRIS se le define en el artículo $3^{\circ}$, fracción I, inciso $\mathrm{n}$ de su reglamento, como la entidad encargada de "ejercer la regulación, control, vigilancia y fomento sanitarios, en materia de efectos nocivos de los factores ambientales en la salud humana"; sin embargo, hasta el momento no ha ejercido acción alguna sobre esta atribución, al menos en lo que se refiere a radiaciones electromagnéticas (COFEPRIS 2004).

Hasta hoy sólo existe una NOM que regula la exposición ocupacional o laboral a RNI. La primera propuesta de regulación realizada en 1993 y publicada en el Diario Oficial de la Federación (DOF) como NOM-STPS-013-1993 Relativa a las condiciones de seguridad e higiene en los centros de trabajo donde se generen radiaciones electromagnéticas no ionizantes, la cual define los límites de la radiación de personas expuestas por su trabajo de manera constante a $10 \mathrm{~W} / \mathrm{m}^{2}$ (STPS 1993).

Esta norma fue objeto de una primera revisión quinquenal por el Comité Consultivo Nacional de Normalización de Seguridad y Salud en el Trabajo, el cual determinó, en coordinación con el comité respectivo de Regulación y Fomento Sanitario de la Secretaría de Salud, una modificación mínima. Para la segunda revisión se solicitó al IMSS un análisis y estudio estadístico de casos referidos a la norma entre 2001 y 2007, que sirvieron de base para ratificar su vigencia en el estado en que se encontraba al determinar que no se había registrado un incremento en los padecimientos relacionados con la exposición a RNI. La última revisión fue realizada en 2012 (STPS 2012).

En este último año se propuso también el anteproyecto para la NOM-SCT-SSA-126 con el objetivo de regular las RNI en el rango de $100 \mathrm{~Hz}$ a $300 \mathrm{GHz}$ (SCT 2012). Sin embargo, el pleno de la Comisión Federal de Telecomunicaciones (COFETEL) no aprobó esta NOM, quedando trunco el 
proceso, ya que dicha Comisión desapareció con la nueva administración federal al reformarse la Ley Federal de Telecomunicaciones y Radiodifusión (LFTR) en 2013.

En 2013, la reforma a la LFTR dio nacimiento al Instituto Federal de Telecomunicaciones (IFT), organismo autónomo que concentró atribuciones de la Secretaría de Comunicaciones y Transportes (SCT) y de la COFETEL con el propósito de promover y reforzar el sector. El artículo 65 de dicha ley otorgó al IFT la atribución de definir los límites de exposición humana a campos electromagnéticos en el rango de los 100 a los $300 \mathrm{GHz}$ en colaboración con otras dependencias. De este mandato nacieron dos propuestas de disposición técnica (DT), la DT-IFT-007 concerniente a exposiciones de campo lejano (antenas, por ejemplo) y la DTIFT-012 referente a exposiciones de campo cercano (celulares, ruteadores inalámbricos). Sin embargo, estos procesos, contemplados en los planes anuales de trabajo del IFT desde 2015, no se han concluido (IFT 2015, 2018, CDDHCU 2018b).

El cuadro I resume el marco regulatorio en México sobre la emisión y exposición a RNI, y muestra

CUADRO I. RESUMEN DEL MARCO REGULATORIO EN MÉXICO SOBRE LA EMISIÓN Y EXPOSICIÓN HUMANA A RADIACIONES ELECTROMAGNÉTICAS NO IONIZANTES

\begin{tabular}{|c|c|c|c|c|}
\hline Dependencia & Atribución & Sustento jurídico & Cumplimiento & Estatus \\
\hline \multirow[t]{2}{*}{$\begin{array}{l}\text { Secretaría del } \\
\text { Medio Ambiente y } \\
\text { Recursos Naturales }\end{array}$} & $\begin{array}{l}\text { Expedición de NOM y vigilancia } \\
\text { de su cumplimiento }\end{array}$ & $\begin{array}{l}\text { Ley General del } \\
\text { Equilibrio Ecoló- } \\
\text { gico y Protección } \\
\text { Ambiental; artículo } \\
5 \text {, fracción v }\end{array}$ & $\begin{array}{l}\text { Sin Norma Oficial Mexicana (junio } \\
\text { de 2016) }\end{array}$ & $\begin{array}{l}\text { Sin fecha de } \\
\text { inicio de pro- } \\
\text { ceso de NOM }\end{array}$ \\
\hline & $\begin{array}{l}\text { Regular la prevención de la con- } \\
\text { taminación ambiental originada } \\
\text { por radiaciones electromagné- } \\
\text { ticas }\end{array}$ & $\begin{array}{l}\text { Ley General del } \\
\text { Equilibrio Ecoló- } \\
\text { gico y Protección } \\
\text { Ambiental; artículo } \\
5 \text {, fracción XV }\end{array}$ & $\begin{array}{l}\text { Sin Norma Oficial Mexicana (junio } \\
\text { de 2016) }\end{array}$ & $\begin{array}{l}\text { Sin fecha de } \\
\text { inicio de pro- } \\
\text { ceso de NOM }\end{array}$ \\
\hline $\begin{array}{l}\text { Secretaría del Trabajo y } \\
\text { Previsión Social }\end{array}$ & $\begin{array}{l}\text { Normar los tiempos e intensidad } \\
\text { de la exposición ocupacional }\end{array}$ & $\begin{array}{l}\text { Artículos } 2^{\circ}, 3^{\circ}, \text { y } \\
5^{\circ} \text { del Reglamento } \\
\text { General de Segu- } \\
\text { ridad e Higiene en } \\
\text { el Trabajo y } 5^{\circ} \text { del } \\
\text { reglamento interior } \\
\text { de la Secretaría del } \\
\text { Trabajo y Previsión } \\
\text { Social }\end{array}$ & $\begin{array}{l}\text { NOM-013-STPS-1993 Relativa } \\
\text { a las condiciones de seguridad e } \\
\text { higiene en los centros de trabajo } \\
\text { donde se generan radiaciones elec- } \\
\text { tromagnéticas no ionizantes }\end{array}$ & Vigente \\
\hline \multirow[t]{3}{*}{ Secretaría de Salud } & $\begin{array}{l}\text { La prevención y el control de } \\
\text { los efectos nocivos de los facto- } \\
\text { res ambientales en la salud del } \\
\text { hombre }\end{array}$ & $\begin{array}{l}\text { Ley General de } \\
\text { Salud; artículo } 3^{\circ} \\
\text { fracción XIII }\end{array}$ & \multirow{3}{*}{$\begin{array}{l}\text { NOM-048-SSA1-1993, Que es- } \\
\text { tablece el método normalizado } \\
\text { para la evaluación de riesgos a la } \\
\text { salud como consecuencia de agente } \\
\text { ambientales. } \\
\text { A partir de la reforma a la LGS en } \\
\text { 2001, que dio lugar a la creación de } \\
\text { la COFEPRIS, este órgano ejercerá } \\
\text { las facultades que le confiere la ley } \\
\text { en materia de regulación, control } \\
\text { y fomento sanitario, encargándose } \\
\text { también de la elaboración y ex- } \\
\text { pedición de las normas oficiales } \\
\text { (artículo } 17 \text { b) }\end{array}$} & \multirow[t]{3}{*}{$\begin{array}{l}\text { Sin fecha de } \\
\text { inicio de pro- } \\
\text { ceso de NOM } \\
\text { NOM-048 Vi- } \\
\text { gente }\end{array}$} \\
\hline & $\begin{array}{l}\text { La salud ocupacional y el sanea- } \\
\text { miento básico }\end{array}$ & $\begin{array}{l}\text { Ley General de Sa- } \\
\text { lud; artículo } 3^{\circ} \\
\text { fracción XIV }\end{array}$ & & \\
\hline & $\begin{array}{l}\text { Establecer las normas, tomar las } \\
\text { medidas y realizar las actividades } \\
\text { tendientes a proteger la salud } \\
\text { humana ante los riesgos y daños } \\
\text { dependientes de las condiciones } \\
\text { del ambiente }\end{array}$ & $\begin{array}{l}\text { Ley General de } \\
\text { Salud; artículo } 16\end{array}$ & & \\
\hline $\begin{array}{l}\text { Instituto Federal de } \\
\text { Telecomunicaciones }\end{array}$ & $\begin{array}{l}\text { Definir los límites de } \\
\text { exposición humana }\end{array}$ & $\begin{array}{l}\text { Ley Federal de Te- } \\
\text { lecomunicaciones } \\
\text { y Salud, artículo } 65\end{array}$ & $\begin{array}{l}\text { Disposición técnica; límites de } \\
\text { exposición humana }\end{array}$ & $\begin{array}{l}\text { En proceso } \\
(2015)\end{array}$ \\
\hline
\end{tabular}

Fuente: elaboración propia 
las cuatro dependencias involucradas en este tema: la Secretaría del Medio Ambiente y Recursos Naturales (SEMARNAT), la Secretaría del Trabajo y Previsión Social (STPS), la SSA y el IFT; además, describe la atribución para cada uno de ellos, así como el sustento jurídico y el estatus de las normas. Al considerar la importancia de la RNI y su impacto nocivo en el ambiente, cabe resaltar el asombro que causa saber que en el país sólo hay una NOM vigente con relación a este tipo de radiación, y además restringida únicamente al ámbito ocupacional (STPS 1993). La otra norma vigente propone una metodología para evaluar los riesgos de la población abierta ante agentes contaminantes (SSA 1993). Por esta razón se cuenta con una gran oportunidad para actualizar y generar nuevas NOM que regulen los niveles máximos permitidos de exposición a RNI dentro de los contextos actuales de exposición, y en consecuencia, propicien la vigilancia y el control de los efectos a la salud.

\section{LEUCEMIA}

La leucemia es una enfermedad caracterizada por detención en la maduración de las células encargadas de la formación de los constituyentes de la sangre, con proliferación y crecimiento descontrolado de células sanguíneas inmaduras. Esta proliferación se origina a nivel de la médula ósea, a partir de la cual se disemina a la sangre y los distintos tejidos.

La leucemia es el tipo más común de cáncer infantil, y representa el $30 \%$ de todos los cánceres diagnosticados en niños menores de 15 años. De ellos, la leucemia aguda linfoide (LAL) es aproximadamente cinco veces más frecuente que la leucemia aguda mieloide (LAM), y representa aproximadamente el $78 \%$ de todos los diagnósticos de leucemia infantil (Torre et al. 2015).

En 1965, Ager et al. (1965) publicaron la posible asociación entre leucemia aguda (LA) infantil y la influencia de factores ambientales. Desde entonces un incremento en la incidencia de casos de leucemia infantil ha sido informado en Europa, Estados Unidos y México, lo cual se ha hecho evidente mediante la identificación de patrones de agrupación de los casos, asociados con componentes etiológicos ambientales (Draper et al. 2005, McNally y Parker 2006, TlacuiloParra et al. 2017).

La incidencia anual de leucemia infantil durante 2003 se estimó en 4/100 000 habitantes según la OMS. Un estudio realizado en la Ciudad de México durante el periodo 2006-2007, informó una tasa de incidencia anual (TIA) de 5.76 casos/100 000 habitantes, siendo la LAL el tipo más frecuente con el $85.1 \%$ de los casos (TIA: 4.95/100 000), seguido de la LAM con el $12.3 \%$ (TIA: 0.69/100 000) (PérezSaldívar et al. 2011). De forma similar, un estudio realizado en el área metropolitana de Guadalajara en 2015 demostró una incidencia global de leucemia infantil de 6.44 casos/100 000 habitantes, la tasa más alta se observó en el municipio de El Salto, con 10.12 casos/100000 habitantes, seguido de Guadalajara con 7.55/100 000 habitantes, y Tlaquepaque con 6.74 casos/100 000 habitantes (Soto-Sumuano et al. 2016, Tlacuilo-Parra et al. 2017).

\section{RADIACIÓN ELECTROMAGNÉTICAY LEUCEMIA}

Los primeros trabajos sobre la asociación entre el campo magnético de las líneas de alta tensión y baja frecuencia (FEB) y leucemia aparecieron en 1930 en EUA, cuando se detectó un incremento en la tasa de incidencia anual de leucemia en todos los estados donde se había introducido la electricidad en más del $75 \%$ de las residencias (Milham et al. 2001). Para 1950, la incidencia elevada se mantenía en todos los estados, aunque en comparación era más pronunciada en aquellos con mayor porcentaje de contactos intradomiciliarios a la red eléctrica. Según Milham y Ossiander (2001), la asociación de leucemia con urbanización, modernización e industrialización puede explicarse por el incremento en la electrificación. Koifman (1993) sugirió a su vez que la RNI podía ser carcinogénica, propuesta que fue apoyada por los hallazgos de la publicación Estudio de Investigación del Cáncer Infantil en Reino Unido (UKCCS 1999). Sin embargo, fue hasta después de la publicación del análisis agrupado de Ahlbom et al. (2000) cuando la IARC comenzó a considerar a la RNI de FEB como posible carcinogénico.

Los estudios mencionados refieren un incremento del riesgo de contraer leucemia pediátrica cuando los niveles de radiación en FEB son mayores a $0.3 \mu \mathrm{T}$ (micro-Teslas) (Ahlbom et al. 2000, Greenland et al. 2000, Calvente et al. 2010, Kundi 2012).

Considerando el estudio realizado en Guadalajara por Tlacuilo-Parra et al. (2017), donde se demostró la presencia de focos de agrupación geográfica de niños con leucemia aguda (LA), el Instituto Mexicano del Seguro Social (IMSS) propuso realizar un estudio en su Hospital de Pediatría del Centro Médico Nacional de Occidente y el Nuevo Hospital Civil de Guadalajara, con la finalidad de determinar el nivel 
de exposición a radiación electromagnética en FEB (centrada a $60 \mathrm{~Hz}$ ) y RF (en un rango de $100 \mathrm{KHz}$ a $5 \mathrm{GHz}$ ) intradomiciliaria de niños con leucemia y proponer un plan de prevención y observación de dicha radiación.

\section{MATERIALES Y MÉTODOS}

Se incluyeron pacientes con diagnóstico de LA infantil atendidos en el Hospital de Pediatría del Centro Médico Nacional de Occidente (CMNO) perteneciente al IMSS y del Nuevo Hospital Civil de la Universidad de Guadalajara que habitaron en la zona metropolitana de Guadalajara durante el periodo 2013-2016.

El paciente incluido en el estudio debía cumplir con los criterios siguientes: ser menor de 16 años, vivir en la zona metropolitana en la misma casa al menos por un año antes del diagnóstico, el diagnóstico de LA tenía que ser confirmado por aspiración de médula ósea y por inmunotipificación, y haber sido tratado en cualquiera de los hospitales mencionados durante el periodo del estudio.

De los 84 casos elegibles, el $4 \%$ rechazó el estudio, pero el $96 \%$ ingresó con éxito. Se identificaron mediante encuesta las fuentes de RNI divididas en fuentes extradomiciliarias e intradomiciliarias, y se acudió al domicilio del paciente para medir los niveles de radiación en FEB, así como en RF.

\section{Equipo y medición de la radiación en el rango de FEB y RF}

Las mediciones se realizaron en la cama de los pacientes dentro de sus casas, donde pasan $8 \mathrm{~h}$ continuas, para lo cual se utilizaron:

- Medidor portable HI-3604 ETS-Lindgren para medir la intensidad de campo magnético en $\mu \mathrm{T}$ (micro-Teslas) en el rango de las frecuencias de FEB, con antena sintonizada a $60 \mathrm{~Hz}$.

- Equipo portable HI-2200 para mediciones de RF de intensidad de campo eléctrico en volts $/ \mathrm{m}$ y densidad de potencia en $\mathrm{W} / \mathrm{m}^{2}$, con antena para un rango de frecuencias desde los $100 \mathrm{KHz}$ hasta los $5 \mathrm{GHz}$.

El protocolo para dichas mediciones fue el propuesto por el Instituto de Ingenieros Eléctricos y Electrónica (IEEE 1992), y el proyecto de investigación fue aprobado por los comités de ética e investigación de los hospitales participantes. En un periodo de 12 meses, se visitó el domicilio de los pacientes hasta en cuatro ocasiones para corroborar datos y minimizar cualquier posible error de medición.

Para la estadística descriptiva se emplearon valores como el promedio, mediana y desviación estándar. Para la estadística inferencial se empleó la prueba t de Student para comparar promedios entre hospitales y la prueba chi-cuadrada para comparar variables cualitativas.

\section{RESULTADOS}

Participaron 80 pacientes, 72 con diagnóstico de LAL $(90 \%)$ y 8 con LAM (10 \%), 43 masculinos (54\%) y 37 femeninos (46\%). Su edad promedio al momento de diagnóstico fue de $6 \pm 4$ años, y al momento del estudio $9 \pm 4$ años.

El nivel de exposición promedio para FEB en las camas de los pacientes fue de $0.25 \pm 0.35 \mu \mathrm{T}$ (mediana $0.09 \mu \mathrm{T}$ ). En el $32 \%$ de los casos se encontraron niveles superiores al punto de corte recomendado $(0.3 \mu \mathrm{T})$. No hubo diferencias significativas entre el promedio y las medianas obtenida en las casas, entre pacientes de ambos hospitales: $0.28 \pm 0.37 \mu \mathrm{T}$ (mediana $0.13 \mu \mathrm{T}$ ) vs $0.19 \pm 0.28 \mu \mathrm{T}$ (mediana 0.08 $\mu \mathrm{T})(\mathrm{p}=0.251)$. Cuando se categorizó en grupos para valores $\geq 0.3 \mu \mathrm{T}$, el $32 \%$ ( 26 casos) de las mediciones fueron valores más altos que el nivel de referencia y el $5 \%$ de los casos obtuvo valores mayores a 1.0 $\mu \mathrm{T}$. Las fuentes de RNI en FEB identificadas fueron transformadores eléctricos, líneas de alta tensión, televisores y electrodomésticos.

Para las mediciones en el rango de RF, el nivel de exposición promedio fue de $0.142 \pm 10.3 \mathrm{~W} /$ $\mathrm{m}^{2}$ (mediana $0.54 \mathrm{~W} / \mathrm{m}^{2}$ ). En el $35 \%$ de los casos se encontraron niveles superiores al punto de corte recomendado de $0.1 \mathrm{~W} / \mathrm{m}^{2}$. No hubo diferencias significativas entre el promedio y las medianas obtenida de las casas entre los pacientes de ambos hospitales: $0.075 \pm 0.37 \mathrm{~W} / \mathrm{m}^{2}$ (mediana $0.04 \mathrm{~W} / \mathrm{m}^{2}$ ) vs $0.17 \pm$ $0.28 \mathrm{~W} / \mathrm{m}^{2}$ (mediana $\left.0.05 \mathrm{~W} / \mathrm{m}^{2}\right)(\mathrm{p}=0.583)$. Las fuentes emisoras de RNI de RF identificadas fueron antenas de telefonía celular, hornos de microondas, teléfonos celulares y módems inalámbricos. Es importante señalar que en 11 pacientes $(13 \%)$ se observó exposición a niveles de radiación no ionizante en ambas frecuencias por arriba de lo permitido.

\section{DISCUSIÓN}

Los resultados muestran que un tercio de los niños con leucemia de la zona metropolitana de 
Guadalajara se encuentran expuestos a niveles altos de FEB y RF. De acuerdo con la literatura, los niveles observados de exposición en FEB (corte a $0.3 \mu \mathrm{T}$ ) les confieren un riesgo dos veces mayor para desarrollar leucemia en comparación con niños no expuestos a dichos niveles de radiación. Se propone como modelo el principio precautorio y se recomiendan acciones prácticas que no generan ningún costo al paciente $\mathrm{y}$ que conducen a disminuir la exposición a RNI (Draper et al. 2005). Un ejemplo reciente de la aplicación del principio precautorio ocurrió en abril de 2019, cuando la Agencia Nacional de Seguridad Sanitaria, Alimentación, Medio Ambiente y del Trabajo de Francia (ANSES, por sus siglas en francés) reiteró sus indicaciones emitidas en 2010 de limitar el número y la exposición de personas sensibles expuestas a líneas de alto voltaje, para lo cual recomendó no construir nuevas instalaciones o ampliar las existentes en sitios donde se atienda a personas sensibles (hospitales, escuelas, etc.) en las inmediaciones de líneas de muy alto voltaje, ni establecer nuevas líneas por encima de dichos establecimientos. Además, la agencia subraya la necesidad de controlar mejor la exposición en el lugar de trabajo para ciertos profesionales que pueden estar expuestos a altos niveles de campos electromagnéticos, en particular mujeres embarazadas (ANSES 2019).

Respecta de los efectos de la RNI ocasionados por RF, si bien no se ha establecido una asociación con LA tan clara como en el caso de la RNI de FEB, sí hay una amplia lista de padecimientos que pueden empeorar la condición física y mental del paciente, entre ellos: problemas cardiacos, de sueño, memoria, aprendizaje, fatiga y comportamiento, presión sanguínea, efectos vasculares, trastorno en el metabolismo del calcio, estrés oxidativo y de proteínas, problemas reproductivos, posible daño y problemas de reparación en el ADN (ácido desoxirribonucleico), aumento en la posibilidad de tumores cerebrales y permeabilidad de la barrera hematoencefálica (BR 2012).

El principio de precaución es una política de gestión de riesgos que se aplica en circunstancias de incertidumbre científica, la cual refleja la necesidad de tomar medidas para un riesgo potencialmente grave sin esperar los resultados de la investigación. Para los países de la Unión Europea, el Tratado de Roma (1957) establece que "...la política comunitaria sobre el medio ambiente se basará en el principio de precaución" (PE 1957). Otro enfoque existente se denomina As Low As Reasonably Achievable (ALARA), traducido al español como tan bajo como sea razonablemente posible y funcional. Los principios
ALARA son impulsados por el Parlamento Europeo con sustento en la Resolución 1815 (CE 2011). Un ejemplo reciente de adopción del principio ALARA es la decisión de algunos países europeos de reducir los niveles en entornos con presencia humana prolongada (igual o mayor a $4 \mathrm{~h}$ ) o en puntos sensibles como hospitales, asilos, edificios públicos, edificios turísticos, escuelas, guarderías, jardines de niños, parques y áreas de recreación. Algunos países con tales disposiciones son Italia, Grecia, Bélgica, Eslovenia y Polonia.

El crecimiento acelerado que ha experimentado la industria de las telecomunicaciones en los últimos años en México ha sido un éxito por su impacto social y económico, colocando al país en un nivel privilegiado en la oferta de servicios. Sin embargo, existe un rezago muy importante en la regulación y control de los niveles de emisión y exposición a las RNI, por lo cual es urgente que las entidades de gobierno involucradas en la salud de la población emprendan acciones al respecto.

Se ha descrito que a nivel mundial existe una preocupación sobre el efecto de las RNI y su asociación con enfermedades, sin embargo y como ya se mencionó anteriormente en México sólo hay una NOM vigente, restringida al ámbito ocupacional (STPS 1993), y otra NOM que propone una metodología para evaluar los riesgos de la población abierta ante agentes contaminantes (SSA 1993). Existe la necesidad imperante de una NOM para la regulación de la exposición al público en general. Sólo hubo un intento de regulación que creó un anteproyecto de NOM promovido por la SCT que nunca se concretó (SCT 2012).

Asimismo, se debe analizar cabalmente a quién le corresponde fijar los límites de exposición humana a las RNI siendo ésta un riesgo de salud pública y no un factor técnico.

Según lo establecido en el art. 28 de la Constitución Política de Estados Unidos Mexicanos, el IFT “...tendrá a su cargo la regulación, promoción y supervisión del uso, aprovechamiento y explotación del espectro radioeléctrico, las redes y la prestación de los servicios de radiodifusión y telecomunicaciones, así como del acceso a infraestructura activa, pasiva y otros insumos esenciales, garantizando lo establecido en los artículos 6o. y 7o. de esta Constitución" (CDDHCU 2018a). El hecho que un tema tan importante como el descrito en este documento sea encabezado por un organismo cuya prioridad son los proyectos de infraestructura tecnológica en telecomunicaciones, es un obstáculo para una regulación que debe cumplir con criterios de sa- 
lud pública. Es imprescindible destacar que en la población general se desconoce el efecto adverso y el peligro que representan los altos niveles de exposición a RNI, y al no existir registros públicos de mediciones realizadas por ningún ente público de salud, ambiental o de telecomunicaciones, aumenta la incertidumbre respecto a que se hayan rebasado o no las recomendaciones internacionales en los últimos 22 años cuando nos referimos a los límites fijados en 1996 por la FCC.

Se ha identificado un gran desconocimiento de la problemática de la RNI y de las normas y recomendaciones internacionales vigentes por parte de las autoridades estatales y municipales. Hasta el momento, los gobiernos municipales sólo se han involucrado en el proceso administrativo y cobro de los permisos de instalación de la estructura donde se colocarán las antenas.

Un ejemplo de lo anterior es la instalación de infraestructura de telecomunicaciones para la telefonía móvil en uso de suelo vecinal y mixto barrial en Jalisco. En ambos usos de suelo, los servicios de telecomunicaciones no están permitidos (GJ 2011); sin embargo, donde sí se permite instalar servicios de telecomunicaciones hay múltiples instalaciones irregulares, (Figs. 2 y 3), lo que aumenta la posibilidad de exposición a altos niveles de radiación en espacios sensibles.

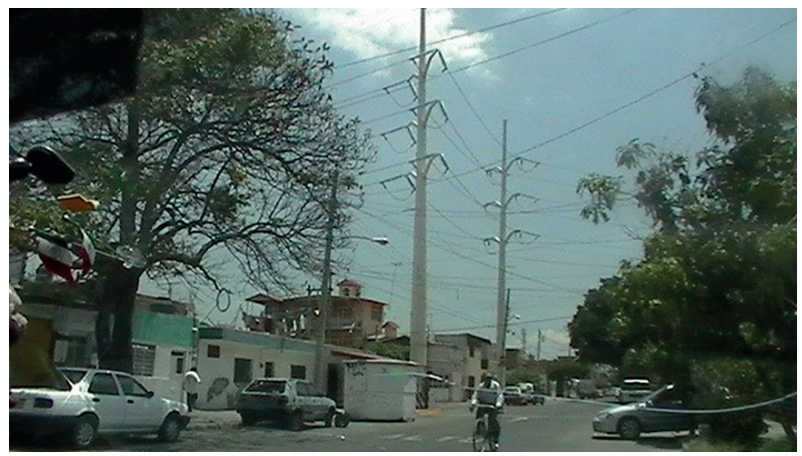

Fig. 2. Distribución de energía eléctrica de alta tensión en zonas urbanas

Se propone la introducción de criterios en la planeación del desarrollo urbano que respeten el equilibrio ambiental y propicien una relación armónica de la población con el medio ambiente; sin embargo, debido a la necesidad de acelerar la modernización de las telecomunicaciones, no se contemplaron aspectos regulatorios relativos a los niveles de emisión y exposición a la RNI y su posible impacto a la salud.

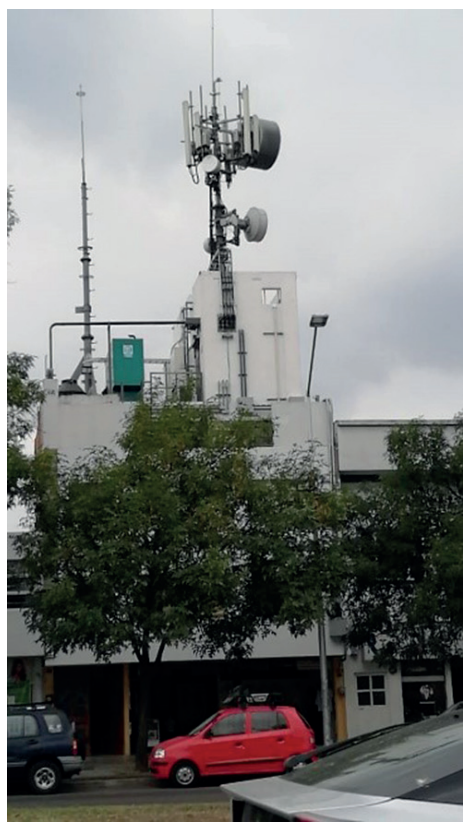

Fig. 3. Antenas de telefonía celular a baja altura

\section{CONCLUSIONES}

Como se describe anteriormente, el artículo muestra un escenario inquietante de las emisiones de RNI en México. Las mediciones realizadas en los diferentes domicilios confirman la existencia de valores muy altos de radiación tanto en baja como en alta frecuencia comparados con los umbrales establecidos en otros países, lo cual nos invita a reflexionar en la existencia de un factor asociado a mayor riesgo para el desarrollo de enfermedades como leucemia, por lo cual la radiación se manifiesta como un contaminante importante. Asimismo, la necesidad de establecer normas para niveles máximos de exposición al público en general se hace imperativa ante la gran cantidad de estudios que argumentan la existencia de una posible causalidad de los efectos negativos de la RNI en la salud pública.

La ausencia de una NOM que regule la exposición del público en general a RNI ha propiciado la ausencia de registros de niveles de contaminación electromagnética. Por ello se propone que las nuevas regulaciones incluyan el monitoreo constante de los niveles de RNI, sobre todo en zonas sensibles (hospitales, asilos, guarderías, etc.). También se propone que se definan nuevas normas tomando en cuenta los niveles actuales de exposición, ya que las recomendaciones actuales datan de finales de la década de 1990, cuando la exposición era muy baja en comparación con la actual. Las nuevas tecnologías, como la $5 \mathrm{~g}$, incrementarán 
de manera exponencial la exposición del público en general, principalmente por la instalación de antenas a distancias muy cortas (de 50 a $150 \mathrm{~m}$, dependiendo de la frecuencia y la cantidad de conexiones) y casi a nivel de piso en postes o sobre fachadas para estar cerca de los usuarios y de los objetos que se conectarán para el llamado internet de las cosas, así como de los vehículos autónomos. Cabe mencionar que existe la posibilidad de relajar los niveles máximos recomendados, pues se ha reconocido que éstos pueden significar un problema para el despliegue comercial de las redes $5 \mathrm{~g}$ (Törnevik 2017).

Es imperativo investigar si el incremento en los casos de leucemia aguda en niños en los hospitales de pediatría del país se debe a la contaminación ambiental o a otras razones. Por ello se propone un estudio para medir el nivel de exposición a RNI de pacientes con leucemia aguda en todo el país, así como en escuelas de educación básica y puntos sensibles. Para procesar y mostrar los datos obtenidos de las mediciones, se propone utilizar una aplicación desarrollada en el IMSS, denominada SirGeoS, que despliega, de manera georreferenciada, los contaminantes presentes en las zonas donde se ubican pacientes con LA y cualquier otro padecimiento que pudiera tener asociación con RNI de FEB o RF (Soto-Sumuano et al. 2017), considerando estudios recientes sobre hematología geográfica de México (Colunga-Pedraza et al. 2018).

Adicionalmente, se ha detectado falta de orden urbano, factor que ha incrementado la exposición no laboral. Por un lado, se encontraron casas muy cerca de fuentes de emisión de alto voltaje (a menos de $10 \mathrm{~m}$ de líneas de alta tensión) (Fig. 2) o bien que colindan con los nodos de distribución de energía eléctrica de la Comisión Federal de Electricidad. Por otro lado, en el caso de mástiles (donde se colocan equipos y antenas) se observaron antenas de telefonía celular colocadas sin la altura adecuada, muchas a una altura menor a $5 \mathrm{~m}$, y otras sobre casas habitación en total desapego con lo referido en las normas internacionales que proponen colocarlas al menos a una altura de $30 \mathrm{~m}$ del suelo. (Fig. 3)

Es importante que se reformen las leyes involucradas en este tema, para que se generen las normas oficiales que aporten guía y seguridad en el control de las emisiones, ya que su ausencia afecta la salud de la población infantil al permitir la exposición a dosis excesivas de RNI, como consecuencia del crecimiento en la infraestructura radiante. Ante ello, otros países como Francia, Alemania y Holanda han regulado y disminuido los niveles de exposición permitidos a la sociedad en conjunto (CE 2011).
Las reflexiones aquí expresadas pretenden concientizar acerca de la importancia que tiene el desarrollo de las telecomunicaciones, siempre y cuando éste se logre sin afectar al medio ambiente $y$, en consecuencia, a la salud humana. Por último, se recomienda a los organismos gubernamentales, generar campañas de información y concientización pública sobre el uso de equipos que emitan radiación electromagnética y cuidar la cercanía a fuentes de radiación intensa.

Como conclusión general, el reto consiste precisamente en encontrar la fórmula para conciliar el crecimiento de las telecomunicaciones sin menoscabo de la salud humana.

\section{AGRADECIMIENTOS}

El presente trabajo fue realizado con el apoyo del Instituto Mexicano del Seguro Social, el Hospital Civil de Guadalajara y la Universidad de Guadalajara, a los cuales expresamos nuestro profundo reconocimiento.

\section{REFERENCIAS}

Ager E.A., Schuman L.M., Wallace H.M., Rosenfield A.B. y Gullen W.H. (1965). An epidemiological study of childhood leukemia. J. Chronic Dis. 18 (2), 113-132. DOI: 10.1016/0021-9681(65)90096-2

Ahlbom A., Day N., Feychting M., Roman E., Skinner J. y Dockerty J. (2000). A pooled analysis of magnetic fields and childhood leukaemia. Brit. J. Cancer 83, 692-698. DOI: 10.1054/bjoc.2000.1376

ANSES (2019). Effets Sanitaires liés à l'exposition aux champs electromagnétiques basses fréquences. Reporte. Agence Nationale de Sécurité Sanitaire de l'Alimentation, de l'Environnement et du Travail. París, Francia, 298 pp.

BR (2012). A rationale for biologically based public exposure standards electromagnetic fields (ELF and RF). Bioinitiative Report [en línea]. https://bioinitiative. org/ 15/08/2018.

Cabañas E.M. (2017). La exposición a las radiaciones electromagnéticas de origen tecnológico: un problema de salud pública mundial. MedLab 9 (1), 11-24.

Calvente I., Fernández M.F., Villalba J., Olea N. y Núñez M.I. (2010). Exposure to electromagnetic fields (nonionizing radiation) and its relationship with childhood leukemia: A systematic review. Sci Total Environ. 408 (16), 3062-3069.

DOI: 10.1016/j.scitotenv.2010.03.039 
CDDHCU (2018a). Constitución Política de los Estados Unidos Mexicanos. Cámara de Diputados del Honorable Congreso de la Unión. Jurídico. Ciudad de México, México, 314 pp.

CDDHCU (2018b). Ley Federal de Telecomunicaciones y Radiodifusión. Cámara de Diputados del Honorable Congreso de la Unión. Jurídico. Ciudad de México, México, $151 \mathrm{pp}$.

CDDHCU (2018c). Ley General de Equilibrio Ecológico y Protección Ambiental (LGEEPA). Cámara de Diputados del Honorable Congreso de la Unión. Jurídico. Ciudad de México, México, 135 pp.

CDDHCU (2018d). Ley General de Salud (LGS). Cámara de Diputados del Honorable Congreso de la Unión. Jurídico. Ciudad de México, México, 303 pp.

CE (2011). The potential dangers of electromagnetic fields and their effect on the environment. Council of Europe [en línea]. https://assembly.coe.int/nw/xml/XRef/XrefXML2HTML-en.asp?fileid=17994 10/10/2018.

COFEPRIS (2004). Reglamento de la Comisión Federal para la Protección contra Riesgos Sanitarios. Comisión Federal para la Protección contra Riesgos Sanitarios. Ciudad de México, México 15 pp.

Colunga-Pedraza P.R., Gómez-Cruz G.B., ColungaPedraza J.E. y Ruiz-Argüelles G. (2018). Geographic hematology: Some observations in Mexico. Acta Haematol. 140 (2), 114-120. DOI: 10.1159/000491989

CS (1999). Ordennance sur la protection contre le rayonnement non ionisant. Confédération Suisse, Conseil Générale [en línea]. https://www.admin.ch/opc/fr/classified-compilation/19996141/index.html 15/10/2018.

Draper G., Vincent T., Kroll M.E. y Swanson J. (2005). Childhood cancer in relation to distance from high voltage power lines in England and Wales: A casecontrol study. Brit. Med. J. 330 (7503), 1290-1294. DOI: $10.1136 / \mathrm{bmj} .330 .7503 .1290$

FCC-OET (1997). Bulletin 65: Evaluating compliance with FCC guidelines for human exposure to radiofrequency electromagnetic fields. Boletín. Federal Communications Commission - Office of Engineering and Technology. Washington, D.C., EUA, 84 pp.

GJ (2011). Reglamento estatal de zonificación de Jalisco. Reglamento. Gobierno de Jalisco, Guadalajara, México, 224 pp.

Greenland S., Sheppard A.R., Kaune W.T., Poole C. y Kelsh M.A. (2000). A pooled analysis of magnetic fields, wire codes, and childhood leukemia. Childhood leukemia EMF study group. Epidemiology 11 (6), 624-634.

DOI: 10.1097/00001468-200011000-00003

IARC (2002). Non-ionizing radiation, part 1: Static and extremely low-frequency (ELF) electric and magnetic fields. Vol. 80. Monografía. International Agency for Research on Cancer. Lyon, Francia, 445 pp.
IARC (2011). Non-ionizing radiation, part 2: Radiofrequency electromagnetic fields. Vol. 102. Monografía. International Agency for Research on Cancer. Lyon, Francia, $480 \mathrm{pp}$.

ICNIRP (2009). Guidelines for limiting exposure to timevarying electric, magnetic, and electromagnetic fields (up to $300 \mathrm{GHz}$ ). International Commission on NonIonizing Radiation Protection [en línea]. https://www. icnirp.org/cms/upload/publications/ICNIRPemfgdl. pdf 15/10/2018.

ICNIRP (2010). Guidelines for limiting exposure to timevarying electric and magnetic fields $(1 \mathrm{~Hz}-100 \mathrm{kHz})$. International Commission on Non-Ionizing Radiation Protection [en línea]. https://www.icnirp.org/cms/ upload/publications/ICNIRPLFgdl.pdf 15/10/2018.

IEEE (1992). Standard for safety levels with respect to human exposure to radio frequency electromagnetic fields, $3 \mathrm{kHz}$ to $300 \mathrm{GHz}$. Institute of Electrical and Electronic Engineers. Manual. Nueva York, EUA, 71 pp. DOI: 10.1109/IEEESTD.1992.101091

IFT (2015). Programa anual de trabajo 2015. Manual. Instituto Federal de Telecomunicaciones. Ciudad de México, México, 40 pp.

IFT (2018). Programa anual de trabajo del Instituto Federal de Telecomunicaciones 20Cd. 18. Manual. Instituto Federal de Telecomunicaciones. Ciudad de México, México, 298 pp.

Koifman S. (1993). Electromagnetic fields: a cancer promoter? Med. Hypotheses 41 (1), 23-27. DOI: 10.1016/0306-9877(93)90027-N

Kundi M. (2012). Evidence for childhood cancers (leukemia) [en línea]. http://www.bioinitiative.org/ 20/06/2018.

McNally R.J. y Parker L (2006). Environmental factors and childhood acute leukemias and lymphomas. Leuk. Lymphoma 47 (4), 583-598

DOI: $10.1080 / 10428190500420973$

Milham S. y Ossiander E.M. (2001). Historical evidence that residential electrification caused the emergence of the childhood leukemia peak. Med. Hypotheses 56 (3), 290-295. DOI: 10.1054/mehy.2000.1138

PE (1957). Tratado de Roma de la Comunidad Económica Europea. Parlamento Europeo [en línea]. http:// www.europarl.europa.eu/about-parliament/es/in-thepast/the-parliament-and-the-treaties/treaty-of-rome 10/07/2018.

Pérez-Saldívar M.L., Fajardo-Gutiérrez A.F., BernáldezRíos R, Martínez-Avalos A., Medran-Sanson A., Espinosa-Hernández L., Flores-Chapa J.D., AmadorSánchez R., Peñaloza-González J.G., ÁlvarezRodríguez F.J., Bolea-Murga V., Flores-Lujano J., Rodríguez-Zepeda M.C., Rivera-Luna R., DorantesAcosta E., Jiménez-Hernández E., Alvarado-Ibarra M., 
Torres-Nava J.R., Duárte-Rodríguez D.A., ParedesAguilera R., Campo-Martínez M.A., Cárdenas-Cardos R., Alamilla-Galicia P.H., Bekker-Méndez V.C., Ortega-Álvarez M.C. y Mejía-Arangure J.M. (2011). Childhood acute leukemias are frequent in Mexico City: Descriptive epidemiology. BMC Cancer 11, 355. DOI: $10.1186 / 1471-2407-11-355$

SCT (2012). Anteproyecto de Norma Oficial Mexicana NOM-126-SCT1-SSA1-2012. Define las medidas de operación para el cumplimiento de los límites de exposición máxima para seres humanos a radiaciones electromagnéticas de radiofrecuencia no ionizantes en el intervalo de $100 \mathrm{kHz}$ a $300 \mathrm{GHz}$ en el entorno de emisores de radiocomunicaciones. Secretaría de Comunicaciones y Transporte, Comisión Federal de Telecomunicaciones, 22 de agosto.

SCT (2017). ¿Qué es México Conectado? Lineamientos. Secretaría de Comunicaciones y Transportes (SCT) [en línea].http://mexicoconectado.gob.mx/sobre_mexico_ conectado.php?id=212 15/12/2017.

Singh S. y Kapoor N. (2014). Health implications of electromagnetic fields, mechanisms of action, and research needs. Adv. Biol. 2014, 24. DOI: 10.1155/2014/198609

Soto-Sumuano J.L., Tlacuilo-Parra J.A., GaribaldiCovarrubias R., Romo-Rubio H. y Arriaga-Dávila J. (2016). Childhood acute leukemia and their association with the high voltage network in Guadalajara Mexico. Memorias. Progress in Electromagnetic Research Symposium (PIERS). Shangai, China. 14-16 de agosto. DOI: $10.1109 /$ piers.2016.7735825

Soto-Sumuano J.L., Olivera-Guerrero F.J., Tlacuilo-Parra J.A., Garibaldi-Covarrubias R., Romo-Rubio H. y Abundis-Gutiérrez E. (2017). Geographical Information System for patients, neoplasms and associated. Memorias. International Conference on Computational Science and its Applications. Trieste, Italia, 3 al 6 de julio, 284-298. DOI: 10.1007/978-3-319-62407-5_20
SSA (1993). Norma Oficial Mexicana NOM-048SSA1-1993. Establece el método de normalizado para la evaluación de riesgos a la salud como consecuencia de agentes ambientales. Secretaría de Salud. Diario Oficial de la Federación, 9 de enero.

STPS (1993). Norma Oficial Mexicana NOM-013STPS-1993. Relativa a la exposición humana ocupacional a campos electromagnéticos no ionizantes. Secretaría del Trabajo y Previsión Social. Diario Oficial de la Federación, 6 de diciembre.

STPS (2012). Norma Oficial Mexicana NOM-013STPS-2012. Revisión quinquenal, relativa a la exposición humana ocupacional a campos electromagnéticos no ionizantes. Secretaría del Trabajo y Previsión Social, Dirección General de Seguridad y Salud en el Trabajo. Diario Oficial de la Federación, 6 de agosto.

Tlacuilo-Parra A., Garibaldi-Covarrubias R., Romo-Rubio H., Soto-Sumuano L., Ruiz-Chávez C., Suarez-Arredondo M., Sánchez-Zubieta F. y Gallegos-Castorena S. (2017). Geographical distribution and cluster detection of childhood acute leukemia in the metropolitan area of Guadalajara, Mexico. Rev. Invest. Clin. 69 (3), 159165. DOI: $10.24875 /$ ric. 17002131

Törnevik C. (2017) Impact of EMF limits on 5G network roll-out. Memorias. ITU Workshop on 5g, EMF \& Health. Varsovia, Polonia, 5 al 6 de diciembre. USB.

Torre L.A., Bray F., Siegel R.L., Ferlay J., Lortet-Tieulent J. y Jemal A. (2015). Global cancer statistics. CA Cancer J. Clin. 65 (2), 87-108. DOI: 10.3322/caac.21262

UKCCS (1999). Exposure to power-frequency magnetic fields and the risk of childhood cancer. United Kingdom Childhood Cancer Study Investigators. Lancet 354 (9194), 1925-1931. DOI: 10.1038/sj.bjc.6600602 